\title{
Immediate Newborn Care and Associated Outcomes in the Delivery Room: An Observational Study in Rwanda
}

\author{
Françoise Mujawamariya ${ }^{1 *}$, Fauste Uwingabire ${ }^{1,2}$, Fatuma Murekatete ${ }^{1}$, Pamela Meharry ${ }^{1,3}$
}

${ }^{1}$ School of Nursing and Midwifery, College of Medicine and Health Sciences, University of Rwanda, Kigali, Rwanda 2 Partners in Health, Rwanda

${ }^{3}$ Department of Women's, Children's and Family Health Services, University of Ilinois, Chicago, US

*Corresponding author: Françoise Mujawamariya. School of Nursing and Midwifery, College of Medicine and Health Sciences, University of Rwanda, Huye Campus, Southern Province, Rwanda. Email: francoise.midwife@gmail.com

\begin{abstract}
Background

Neonatal mortality is a worldwide concern, especially in sub-Sahara Africa. Millions of newborn deaths could be prevented with quality care at birth.
\end{abstract}

\section{Objective}

To identify immediate newborn care provided by birth attendants and associated outcomes in the delivery room of a district hospital in Kabgayi, Rwanda.

\section{Methods}

This study used a descriptive cross-sectional design and convenience sampling strategy. The sample of 171 newborns and birth attendants were observed in the delivery room using a standardized checklist during April and May 2019. Descriptive statistics were used to analyze data.

\section{Results}

The majority $(65.5 \%)$ of newborns were female, and at term $(93.6 \%)$. The majority had mouth and nose cleared $(60 \%)$, dried thoroughly $(75.4 \%)$, wet cloth removed $(57.3 \%)$, delayed umbilical cord clamping $(67.3 \%)$, and immediate skin-to-skin contact (67.3\%). Limited interventions included APGAR evaluation $(28 \%)$ and breastfeeding within the first hour (36.6\%). The majority of $135(78.9 \%)$ were stable, and none died.

\section{Conclusion}

The majority of the study population was stable, though all newborns did not benefit from standard immediate newborn care. To reduce neonatal mortality and morbidity in Rwanda, we need to redouble efforts to strengthen the quality of immediate newborn care. Future research is needed to identify effective training to improve newborn outcomes at birth.

Rwanda J Med Health Sci 2020;3(2):238-249

Keywords: Newborns, immediate newborn care, outcomes, Sub-Sahara Africa. 


\section{BACKGROUND}

Globally, millions of newborns die of preventable or treatable conditions in the first month due to a lack of quality care at birth, or skilled care and treatment in the first few days following birth.[1] Newborns predominantly die from preterm birth, infections, and intrapartum-related complications such as asphyxia.[2] Newborns in sub-Saharan Africa (SSA) or Southern Asia are ten times more likely to die in the neonatal period (first 28 days) than those born in high-resource countries.[3] The World Health Organization (WHO) recommends newborn care guidelines for birth attendants (BA) to provide quality newborn care immediately after birth.[4]

Newborn care begins before delivery with the preparation of the environment, including the presence of a skilled birth attendant able to provide basic resuscitation to keep the newborn stable the healthy.[5] At birth, the airway, breathing, and circulation are checked. Stimulation and thermoregulation follow and include thoroughly drying the newborn, removing wet cloths, placing the newborn in skin-to-skin contact (SSC) with the mother, and draping a warm towel over the posterior part of the newborn. Other interventions include delayed umbilical cord clamping, initiation of breastfeeding $(\mathrm{BF})$, and, when needed, a clean surface for resuscitation.[3-4]

However, various studies have shown that the WHO recommendations are not adhered to routinely. A study conducted by De Graft-Johnson et al.,[5] in six African countries, including Ethiopia, Kenya, Madagascar, Mozambique, Rwanda, and Tanzania, found severe gaps in the readiness of health facilities to provide quality postnatal care. The findings showed that among the 2377 newborns, $80 \%$ were dried immediately after birth, meaning $20 \%$ were not, which could lead to hypothermia and less responsiveness to resuscitation if required. Other study findings included $45 \%$ for the overall SSC rate, with only $10 \%$ country-specific for Kenya. Results further revealed that only $43 \%$ initiated BF within the first hour. Early initiation of SSC and early BF significantly contribute to newborn thermal control and infection prevention.[6]

Early BF initiation reduces the risk of neonatal mortality.[7] The findings from research in SSA, including Rwanda [5], demonstrated a critical need for birth attendants to have the necessary knowledge and skills to provide quality newborn care services. Furthermore, health facilities need to be well equipped to enable readiness to save neonatal lives.
The response to reduce neonatal mortality includes the immediate need to "strengthen and invest" in newborn care around the time of birth, and improve the quality and accountability to every newborn.[8] The use of an evidenced-based neonatal protocol helps minimize the neonatal risk of illness and prevention of deaths, [8] and is currently being updated in Rwanda. Despite efforts to reduce neonatal mortality, the 2019 report from the Rwanda Biomedical Centre (RBC) shows a $17 \%$ neonatal death rate.[9] However, the Rwanda Demographic Health Survey (RDHS) reports that $71 \%$ of neonatal deaths are preventable.[10]

According to Khurmi et al.,[11] findings from a Bottleneck Analysis and projections in key maternal and child mortality rates using Lives Saved Tool (LiST) (2000-2015) indicated the "quality of care at birth, especially in newborn health facilities, was missing" in Rwanda. Half of the perinatal deaths (2012-2013) were potentially preventable, and most were stillbirths and early neonatal deaths.[12] Similarly, findings from a study of the six SSA countries during the 2009-2012 period [5] reported mean scores ranging from $36-62 \%$ for health workers' knowledge of immediate newborn care practices. However, health workers in Kenya displayed greater overall knowledge.[5] Since all three studies were conducted during the Millennium Development Goals (MDG) era and included Rwanda, the investigator was curious to determine the current situation in the early years of the Sustainable Development Goals (SDG).

It is vital that we further accelerate progress in newborn health by reducing the neonatal mortality rate to 12 deaths, or less, per 1,000 live births to meet the SDGs by 2030.[13] This study was undertaken to identify immediate newborn care practices provided by birth attendants and associated outcomes in a maternity ward in Rwanda.

\section{METHODS}

\section{Design}

A descriptive cross-sectional study design was used to identify the immediate newborn care provided and associated outcomes in the delivery room at a Kabgayi District Hospital in Rwanda. Kabgayi is a health institution located in the Southern Province. The study was conducted, from April 1 to May 31, 2019.

\section{Participants' recruitment}

The study used a convenience sampling method in order to meet the study objectives. Newborns birthed by spontaneous vaginal deliveries (SVD) in 
the maternity ward at Kabgayi District Hospital while the investigator was present were included. Newborn birthed by cesarean section (C/S), outside the maternity ward of Kabgayi District Hospital or when the investigator was absent were excluded. According to the Hospital report, there was 900 SVD over the previous six months, with an average of 150 per month. Since the data collection was conducted over two months, the study population was 300 . The sample size of 171 was obtained using the Taro Yamane formula.

$n=\frac{N}{1+N(e)^{2}}$

$300: 1.75=171$

\section{Measures}

This study used a tool adapted from the WHO guidelines [3] and the current Rwanda neonatal care protocol (2nd edition).[8] Some items were added to the checklist to meet the study objectives and the local context. Added items included sociodemographic characteristics of the newborn; staff readiness and equipment available before birth; and immediate newborn outcome (stable, unstable newborn - hypothermia, respiratory distress, and demise). The checklist comprised of four sections:

Section one: Demographic characteristics (5 items) included gestational age, mode of delivery, time of birth, time of first cry/breathe, and gender.

Section two: Staff readiness and equipment availability before birth included (4 items) delivery pack, suction and resuscitation materials, emergency trolley, and birth attendants physically prepared. All four items were coded as Yes (1 point) or No (0 points).

Section three: Standardized immediate newborn care provision (14 items) included mouth and nose cleared, newborn dried, wet cloths removed, kept warm and second clean/dry/warm cloth applied, placed in SSC, APGAR evaluated, APGAR documented without evaluation, newborn resuscitated, unnecessary suction, resuscitated in one minute ( $\mathrm{min})$ for those in need, breathing checked, and delayed cord clamping (1-3 minutes). Other provisions included BF within 60 min., or BF after $60 \mathrm{~min}$. All 14 items were scored as Yes (1 point) or No (0 points).

Section four: The section on neonatal outcomes was divided into two parts. a). Immediate newborn care outcomes (3 items) included stable, unstable, and demise; b). Signs of an unstable newborn (3 items) included mild respiratory distress, severe respiratory distress, and hypothermia. The items were scored as, Yes, (1 point) or No (0 points).

\section{Data collection}

The investigator, a registered midwife with ten years of experience working with mothers and newborns, was oriented to the maternity unit's delivery rooms. The investigator observed vaginal deliveries, starting at the second stage of labor and for one hour following the birth. The investigator was aware of being near enough to observe the details of the birth, yet not too close to interfere with the care of the newborn. The care provided to the newborn by the birth attendants, and the stability of the newborn after birth, were observed and recorded (a tick) on the data collection checklist. The investigator kept the checklists with her in a secured container.

\section{Data analysis}

Descriptive statistics were used to calculate frequency distribution and the chi-square $\left(\mathrm{X}^{2}\right)$ test for relationships among variables. A p-value $\leq 0.05$ was considered as having a statistical significance. Data were analyzed using SPSS version 21 .

\section{Ethical considerations}

Approval of the research was obtained from the University of Rwanda, College of Medicine and Health Sciences Institutional Review Board, and the Director-General of the Kabgayi District Hospital. Informed written consent was obtained from all birth attendant participants, and verbal consent to observe the birth was obtained from the mothers. 


\section{RESULTS}

A cross-sectional study design was used to identify the immediate newborn care provided to 171 newborns in the delivery room at Kabgayi District Hospital. The study was conducted from April 1 to May 31, 2019.

\section{Demographic characteristics of newborns}

\section{Table 1. Demographic characteristics of newborns $(n=171)$}

$\left.\begin{array}{ll}\hline \text { Variables } & \mathbf{n}(\%) \\ \hline \begin{array}{l}\text { Mode of delivery } \\ \text { SVD }\end{array} & 171(100.0) \\ \text { Gestation age } & 11(6.4) \\ \text { Preterm } & 160(93.6) \\ \text { Term } & \\ \text { Time of delivery } & 95(55.6) \\ \text { PM } & 76(44.4) \\ \text { First cry } & \\ <5 \text { min } & 113(66.1) \\ >5 \text { min } \\ \text { Gender } \\ \text { Female } \\ \text { Male }\end{array}\right)$

The demographic characteristics of newborns are presented in Table 1 . The majority of newborns were at term $(93.6 \%)$, and female (65.5\%). The majority (55.6\%) was born before noon (AM) and had their first cry in less than 5 minutes after birth $(66.1 \%)$.

\section{Immediate Newborn Care in the Delivery Room}

Table 2. Availability of Materials and Readiness

\begin{tabular}{lll}
\hline Preparation for newborn care & Yes n (\%) & No n (\%) \\
\hline Delivery pack & $155(90.6)$ & $16(9.4)$ \\
BA prepared physical environment & $94(55.0)$ & $77(45.0)$ \\
Suction and resuscitation materials & $16(9.4)$ & $155(90.6)$ \\
Emergency trolley & $5(2.9)$ & $166(97.1)$
\end{tabular}

\section{BA Birth Attendant}

Readiness and availability of resuscitation equipment were observed before the birth, and the results are presented in Table 2 . The majority of births had a delivery pack available (90.6\%), and birth attendants had prepared the physical environment $(55.0 \%)$. However, there were limited suction and resuscitation materials $(9.4 \%)$, and emergency trolley $(2.9 \%)$. 
Table 3. Provided immediate newborn care in the delivery room

\begin{tabular}{lll}
\hline Interventions & $\begin{array}{l}\text { Yes } \\
\mathbf{n}(\%)\end{array}$ & $\begin{array}{l}\text { No } \\
\mathbf{n}(\%)\end{array}$ \\
\hline $\begin{array}{ll}\text { Dried thoroughly } \\
\text { Mouth and nose cleared }\end{array}$ & $129(75.4)$ & $42(24.6)$ \\
Wet cloth removed & $118(69)$ & $53(31)$ \\
APGAR evaluated & $98(57.3)$ & $73(42.7)$ \\
APGAR documented, not evaluated & $49(28.7)$ & $122(71.3)$ \\
Skin to skin contact & $122(71.3)$ & $49(28.7)$ \\
Dry cloth applied & $115(67.3)$ & $56(32.7)$ \\
Cap applied & $88(51.5)$ & $83(48.5)$ \\
Delayed cord clamping & $35(20.5)$ & $136(79.5)$ \\
BA continued checking & $115(67.3)$ & $56(32.7)$ \\
Unnecessary suctioning & $74(43.3)$ & $94(56.7)$ \\
Bag and mask resuscitation & $123(71.9)$ & $48(28.1)$ \\
& $10(5.8)$ & $161(94.2)$
\end{tabular}

\section{BA Birth attendant}

Results of immediate newborn care interventions provided in the delivery room are presented in Table 3 . The majority of newborns were dried thoroughly (75.4\%), mouth and nose cleared (69\%), wet cloth removed $(57.3 \%)$, immediate SSC with mother (63.7\%), covered with a dry cloth $(51.5 \%)$, and had 1-3 min delayed umbilical cord clamping (67.3\%). A limited number were resuscitated with bag and mask (5.8\%). Also, the majority of newborns had the Apgar score estimated and not evaluated (71.3\%), received unnecessary airway clearing $(71.9 \%)$, no cap applied to keep the head warm (79.5\%), and the BA did not continue to check breathing $(56.7 \%)$.

\section{Breastfeeding initiation time in delivery room}

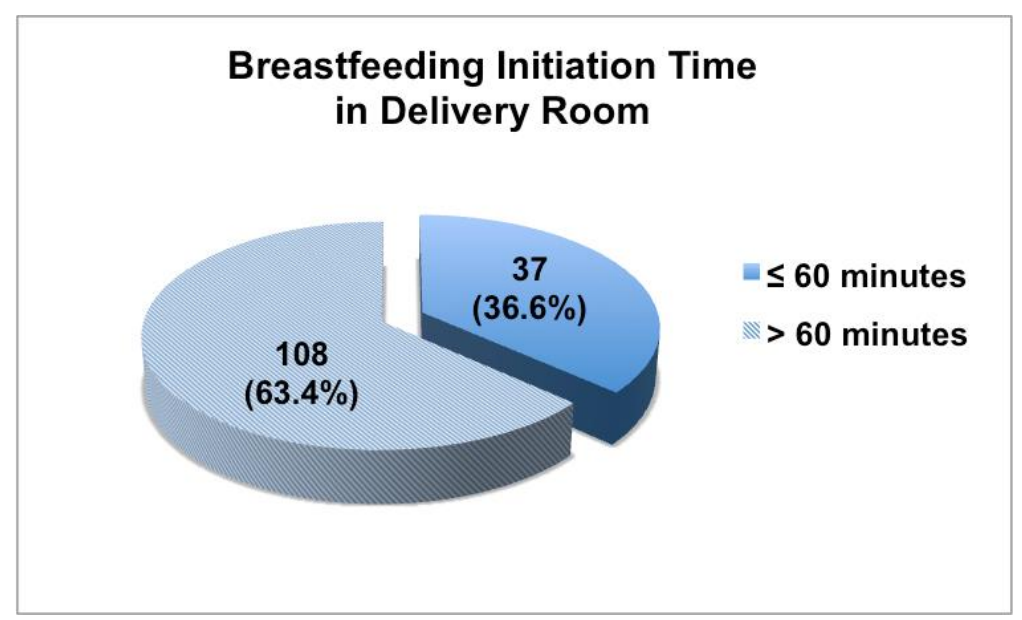

Figure 1. Initial time of breastfeeding in delivery room

The majority of newborns received their initial BF experience in the delivery room, and results are presented in Figure 1. Approximately one third (36.6\%) of newborns initiated BF within 60 min after birth, and two thirds $(63.4 \%)$ initiated $\mathrm{BF}$ after 60 minutes. 


\section{Newborn outcome in delivery room}

\section{Newborn Outcome During Observational Period}

36

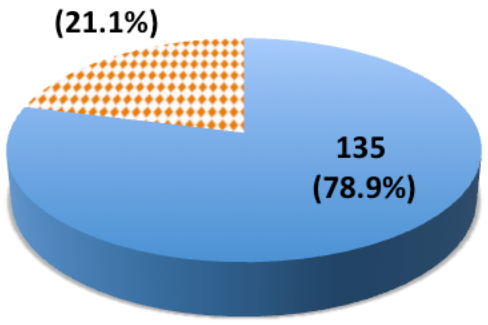

Stable newborn

: Unstable newborn

\section{Figure 2. Newborn outcome in delivery room}

The newborn care and associated immediate outcomes are presented in Figure 2. The majority $(78.9 \%)$ of newborns were stable after birth, whereas nearly a quarter $(21.1 \%)$ was unstable in the hour post-delivery. There was no demise.

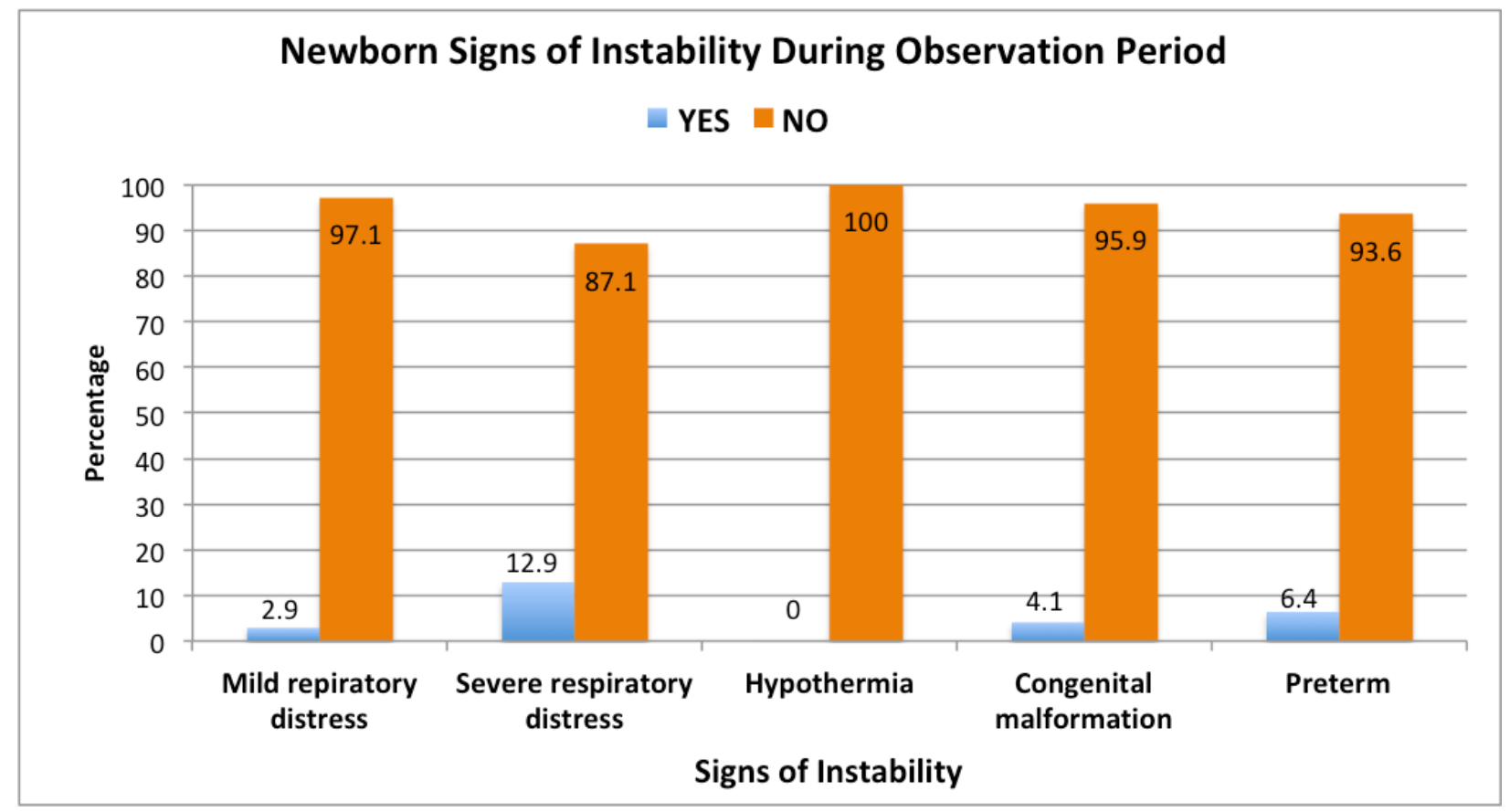

\section{Figure 3. Signs of instability of newborns}

Signs of newborn instability are presented in Figure 3. The newborns that were unstable after birth included a limited number $(2.9 \%)$ who showed signs of mild respiratory distress or severe respiratory distress $(12.9 \%)$. There were no newborns with hypothermia. 
Association between immediate newborn care and immediate outcome

Table 4. Association between immediate newborn care provided and immediate outcome

\begin{tabular}{|c|c|c|c|c|}
\hline \multirow[t]{2}{*}{ Immediate newborn care } & \multicolumn{2}{|c|}{ Immediate outcome } & \multirow[t]{2}{*}{$\mathrm{X}^{2}$} & \multirow[t]{2}{*}{ p-value } \\
\hline & Stable n (\%) & Unstable n (\%) & & \\
\hline \multicolumn{5}{|l|}{ SSC immediate } \\
\hline No & $41(73.2)$ & $15(26.8)$ & \multirow[t]{2}{*}{1.647} & \multirow[t]{2}{*}{0.232} \\
\hline Yes & 94 (81.7) & $21(18.3)$ & & \\
\hline \multicolumn{5}{|l|}{ APGAR score evaluated } \\
\hline No & 94 (77.7) & $27(22.3)$ & \multirow{2}{*}{0.396} & \multirow{2}{*}{0.548} \\
\hline Yes & $41(82.0)$ & $9(18.0)$ & & \\
\hline \multicolumn{5}{|c|}{ APGAR documented without evaluation } \\
\hline No & 39 (79.6) & $10(20.4)$ & \multirow[t]{2}{*}{0.017} & \multirow[t]{2}{*}{0.537} \\
\hline Yes & $96(78.7)$ & $26(21.3)$ & & \\
\hline \multicolumn{5}{|l|}{ Newborn dried thoroughly } \\
\hline No & 39 (92.9) & $3(7.1)$ & \multirow[t]{2}{*}{6.481} & \multirow[t]{2}{*}{$0.007^{*}$} \\
\hline Yes & $96(74.4)$ & $33(25.6)$ & & \\
\hline \multicolumn{5}{|l|}{ Wet cloths removed } \\
\hline No & $52(71.2)$ & $21(28.8)$ & \multirow[t]{2}{*}{4.561} & \multirow[t]{2}{*}{$0.026^{*}$} \\
\hline Yes & $83(84.7)$ & $15(15.3)$ & & \\
\hline \multicolumn{5}{|c|}{ Newborn with 2 nd dry cloth } \\
\hline No & $64(77.1)$ & $19(22.9)$ & \multirow[t]{2}{*}{0.328} & \multirow{2}{*}{0.350} \\
\hline Yes & $71(80.7)$ & 17 (19.3) & & \\
\hline \multicolumn{5}{|c|}{ Newborn kept warm with cap } \\
\hline No & $104(76.5)$ & $32(23.5)$ & \multirow[t]{2}{*}{2.452} & \multirow[t]{2}{*}{0.087} \\
\hline Yes & $31(88.6)$ & $4(11.4)$ & & \\
\hline Mouth and nose cleared, & & & & \\
\hline No & $49(92.5)$ & $4(7.5)$ & 8.429 & $0.002 *$ \\
\hline Yes & $86(72.9)$ & $32(27.1)$ & & \\
\hline Airway had unnecessary & & & & \\
\hline No & $46(95.8)$ & $2(4.2)$ & 11.448 & $<0.00 *$ \\
\hline Yes & 89 (72.4) & $34(27.6)$ & & \\
\hline Timely newborn resuscit & & & & \\
\hline No & $127(78.9)$ & $34(21.1)$ & 0.007 & 0.647 \\
\hline Yes & $8(80.05)$ & $2(20.0)$ & & \\
\hline BA continued to check b & & & & \\
\hline No & 85 (87.6) & $12(12.4)$ & 10.164 & $0.001 *$ \\
\hline Yes & 50 (67.6) & $24(32.4)$ & & \\
\hline Delayed cord clamping & & & & \\
\hline No & $43(76.8)$ & $13(23.3)$ & 0.234 & 0.628 \\
\hline Yes & $92(80.0)$ & $23(20.0)$ & & \\
\hline BR within $60 \mathrm{~min}$ & & & & \\
\hline No & $75(72.1)$ & 29 (27.9) & 7.454 & $0.006^{*}$ \\
\hline Yes & 60 (89.6) & 7 (10.4) & & \\
\hline BR beyond $60 \mathrm{~min}$ & & & & \\
\hline No & $84(82.4)$ & $18(17.6)$ & 1.764 & 0.128 \\
\hline Yes & 51 (73.9) & $18(26.1)$ & & \\
\hline
\end{tabular}

BA Birth Attendant, BR Breastfeeding

There was statistical significance between six immediate newborn care interventions and immediate newborn outcomes, as presented in Table 4. The significant associations included newborn dried thoroughly $(p=0.007)$, wet cloths removed $(p=0.026)$; mouth and nose cleared if meconium $(p=0.002)$; airway had unnecessary suctioning $(p=<0.001)$, BA continued to check breathing $(p=0.001)$; and BF within 60 min $(p=0.006)$.

There was no statistical association with other variables, including SSC $(p=0.232)$, Apgar score evaluated $(p=$ 0.548); APGAR documented without evaluation $(p=0.537)$, newborn with second dry cloth $(p=0.350)$, newborn 
kept warm with cap $(\mathrm{p}=0.087)$, newborn resuscitated on time $(\mathrm{p}=0.647)$; delayed cord clamping $(\mathrm{p}=0.628)$, and $\mathrm{BF}$ beyond $60 \mathrm{~min}(\mathrm{p}=0.128)$.

\section{DISCUSSION}

The study aimed to identify immediate newborn care provided by birth attendants and associated outcomes in the delivery room at a district hospital in Rwanda. Of the 171 newborns that were observed during the first hour, the majority was at term (93.6\%), female (65.5\%), and all were SVD (Table 1). Similarly, a study to improve immediate newborn care practices in hospitals in the Philippines [14], found the majority was at term (98\%), female (66.9\%), and SVD (99\%).

\section{Readiness and availability of materials}

The findings show that the majority of deliveries $(90.6 \%)$ had the delivery pack and the physical environment (55\%) prepared for birth (Table 2$)$. This result is similar to the DeGraf-Johnson study conducted in SSA, whereby $95.4 \%$ had the delivery pack prepared.[5]

Findings in our study that were not so favorable included limited availability of suction and resuscitation materials $(9.4 \%)$ and lack of an emergency trolley (2.9\%). In contrast, suction devices were readily available in $90.7 \%$ of births in DeGraf-Johnson's study in the six-country SSA, [5] and the emergency trolley was ready to roll in $98.7 \%$ of births at the Liverpool Women's Hospital in the UK.[15] In addition to the availability of supplies, other considerations in preparation for every birth include at least one skilled BA in basic resuscitation. A need for resuscitation cannot always be predicted, but a glance at the women's antenatal and labor risk factors could provide some anticipatory guidance for potential resuscitation.[16]

Other studies indicated deficits in essential newborn care supplies. A secondary analysis of the Emergency Obstetric and Newborn Care Assessment in Ethiopia in 2016 found that a quarter of the 3,804 facilities did not have masks in size 0 or 1 in the resuscitation kits.[17] In the sixcountry SSA study, sometimes only a quarter of the needed supplies for immediate newborn care were available. The most significant gaps were in necessary supplies such as towels and blankets, which are essential to dry and drape the newborn to maintain thermoregulation.[5] In Kenya, only $11 \%$ reported all essential equipment was available, which was the highest proportion among the six countries in the SSA study.[5] To prepare for delivery, it is essential to verify that the materials and equipment are intact and functioning well.

\section{Immediate newborn care provided in the delivery room}

\section{Airway and breathing}

Our findings show that the majority of newborns (69\%) had their mouth and nose cleared if meconium was present, though the majority (71.9\%) received unnecessary suctioning of the airway (Table 3). Also, less than half (43.3\%) of BAs continuously checked the newborn's breathing. The study conducted in SSA revealed that only $40 \%$ of newborns with meconium received the correct care.[5] A study in Kenya,[18] found that vital steps in NR were poorly performed, including airway maintenance in the presence of meconium, and ventilation.

In this study, two thirds (66.1\%) had a first cry within five minutes of birth, and of the limited proportion $(5.8 \%)$ of newborns who did not initiate spontaneous breathing, all (100\%) were well resuscitated on time. In contrast to our findings, on the assessment of immediate newborn care, only $42 \%$ of asphyxiated newborns were well resuscitated on time. Findings from a recent retrospective study at a busy district hospital in Rwanda [19] discovered a $39.7 \%$ birth asphyxia rate among NICU admissions. A secondary analysis of national data in Ethiopia [17] found that the newborn survival of asphyxia was increased eightfold with resuscitation, compared to newborns not resuscitated. In the DeGraf-Johnson's sixcountry SSA study, $89 \%$ of newborns that did not cry at birth recovered on their own or through resuscitation.[5]

\section{Delayed cord clamping and cutting}

Our findings showed that over two thirds $(67.3 \%)$ of newborns had delayed cord clamping and cutting at 1-3 minutes, allowing for improved neonatal hemoglobin and hematocrit levels. This finding, therefore, indicates that a third $(32.7 \%)$ had the cord immediately clamped and cut within one minute of birth, which is not the WHO recommendation for best care practice for a stable newborn,[3] though essential for a compromised newborn when resuscitation becomes the priority.[16] A study in a Philippino hospital showed a more favorable rate of $78 \%$ delayed cord clamping.[14] 
A cross-sectional study in Oman of 175 experienced HCPs, including $31 \%$ midwives and $61 \%$ obstetricians found that up to $89.6 \%$ had clamped the cord within one minute of birth for both term and preterm newborns.[20] Interestingly, $45.7 \%$ of the HCPs were concerned with the risk of polycythemia and jaundice of delayed cord care. However, the study also reported the benefits of delayed cord care, namely, allowing the newborn more time for physiological transition to extrauterine life, reduced risk of anemia and increased ferritin, and reduced need for blood transfusions. Moreover, only $11 \%$ of HCPs stated that the timing of cord clamping is guided by hospital policy.[20]

\section{Thermoregulation}

The majority of newborns in our study were dried thoroughly $(75.4 \%)$ to prevent heat loss as the newborn transitions to extrauterine life. Our findings are similar to a study in the Philippines [14] where $80.2 \%$ were dried thoroughly, and the sixcountry SSA study,[5] whereby $79 \%$ of newborns received immediate drying with a cloth or towel. However, many of the newborns with initial breathing difficulties that needed resuscitation were not dried immediately after birth, which increased the risk of hypothermia and decreased the response to resuscitation.[5]

A second systematic review in SSA [21] that involved five countries (Ethiopia, Ghana, Malawi, Tanzania, and Uganda) reported that BAs delayed drying and wrapping the newborn to focus on the mother. The authors of the review reported that this finding was related to the BAs lack of knowledge and practice of routine newborn care, especially immediate SSC with the mother.[5] Thermoregulation is "critical" at birth, as a newborn who is not in SSC with mother or under the warmer can quickly lose heat and spiral downwards, leading to hypoglycemia, acidosis, and potential mortality.[16]

The majority (67.3\%) of newborns in our study were placed in immediate SSC with the mother to minimize heat loss via conduction. Skin-to-skin contact with the mother leads to improved shortterm temperature stability and is better for the newborn than being wrapped in a blanket or placed under the warmer.[22] In contrast, a study conducted in Nigeria, [23] found that only $10-59 \%$ of newborns were placed in SSC immediately after birth. Immediate SSC with the mother is the WHO recommendation for initial thermoregulation that is an affordable and high-quality alternative to technology.[24] Furthermore, if the newborn does develop hypothermia, the first line of treatment is to place the newborn in SSC with the warm mother.[22]
The majority (57.3\%) of newborns in our study had the wet cloth removed, and over half $(51.5 \%)$ were then covered with a second dry cloth. A small proportion $(20.5 \%)$ of newborns had a cap placed on their heads to reduce heat loss. In the SSA study,[5] the wet cloth was removed $57.5 \%$ of the time, while $41 \%$ received a second dry cloth. The systematic review in SSA [21] reported that it was well known that newborns needed to be kept warm, though thermoregulation practice was considered inadequate.

\section{Breastfeeding}

The majority of newborns did not BF within the first hour after birth (63.4\%), though about a third $(36.6 \%)$ did breastfeed in the first hour (Figure 1). These findings may be influenced by the shortage of BAs where the pregnant woman is assisted by one midwife and spends more time caring for the mother, or other mothers, than assisting the newborn to breastfeed. A study found that within the first hour after birth, $43 \%$ of all mothers $(19 \%$ $79 \%$ ) received assistance to initiate $\mathrm{BF}$, and $42 \%$ of mothers in Nigeria and Bangladesh.[23] The SSA study [5] reported that delayed initiation of BF was related to the BA's lack of knowledge and practice. Other barriers include mothers' perception of inadequate milk, newborn not indicating hunger signs, and the newborn needing to sleep.[21]

Early initiation of BF within the first hour after birth has been shown to have positive effects on the newborn, particularly with thermoregulation and infection prevention.[6] All newborns, including preterm who are clinically stable, should be able to $\mathrm{BF}$ as soon as possible after birth if the mother and newborn are willing. $[3,16]$ Breastfeeding significantly decreases infant morbidity and mortality rates, including respiratory diseases, otitis media, gastroenteritis, and diarrhea disorders.[22] All women and their families in low-resource areas need to be giving adequate knowledge to understand the many benefits of beginning breastfeeding within the first hour of birth.[20]

\section{Outcome of immediate newborn care}

This study's findings show that nearly a quarter $(21.1 \%)$ of newborns were unstable within the firsthour post-delivery (Figure 2). The signs of instability were associated with mild respiratory distress $(2.9 \%)$, severe respiratory distress $(12.9 \%)$, preterm $(6.4 \%)$, and congenital malformations (4.1\%) (Figure 3). These findings might be related to the lack of readiness before birth, compared to the WHO recommendations, and that immediate newborn care was started a bit late in some cases. 
A pre-test post-test interventional study conducted by Tran in a tertiary hospital in Da Nang, Viet Nam, revealed the many benefits of implementation of an immediate newborn care program.[25] The interventions demonstrated NICU admissions fell by one third (from $18.3 \%$ to $12.3 \%$ ), hypothermia cases on NICU admissions fell by one quarter (from $5.4 \%$ to $3.9 \%$ ), and sepsis cases in the hospital fell by two thirds (from $3.2 \%$ to $0.9 \%$ ). The intervention in Vietnam,[25] demonstrated that the Early Essential Newborn Care (EENC) initiative included staff training, quality improvement assessments, and changes in the hospital environment and protocols. The results demonstrated improved clinical practices, reduced NICU admissions, reduced newborns with hypothermia and sepsis, and increased exclusive breastfeeding and Kangaroo Mother Care (KMC) in the NICU. If a newborn's temperature is normal (higher than $36.5^{\circ} \mathrm{C}$ ) at the time of admission to the NICU, the duration of mechanical ventilation, length of hospital stay, and mortality rates are decreased.[16] The WHO states that hospitals can reduce life-threatening infections in newborns by over two-thirds and admissions to the NICU by one third by implementing similar initiatives.[26]

\section{Association between immediate newborn outcome and provided care.}

Chi-square analysis showed eight significant associations between immediate care provided to the newborn and immediate newborn outcome (stable or unstable) (Table 4). There was a significant association between immediate newborn outcome and the following interventions: baby dried thoroughly $(\mathrm{p}=0.007)$, wet clothes removed $(\mathrm{p}=$ $0.026)$; mouth and nose cleared if meconium ( $\mathrm{p}=$ $0.002)$; continued to check breathing $(\mathrm{p}=0.001)$, and unnecessary cleaning and suctioning of the airway $(\mathrm{p}=<0.001)$. Further statistical associations with newborn outcome and breastfeeding included initiation within 60 minutes $(p=0.006)$ of birth. There were no statistical associations $(p=>0.05)$ with delayed cord clamping, Apgar score evaluated and documented, newborn resuscitated on time, SSC, wrapped in a second dry cloth, head cap, and breastfeeding beyond 60 minutes.

\section{Limitations}

Data were collected from only one hospital; therefore, the results cannot be generalized to other hospitals in Rwanda. Factors contributing to the provision of immediate newborn care were not studied. Thus, a study is needed to identify challenges associated with immediate newborn care provision by health care providers from the current study setting.

\section{Recommendations}

The BAs in maternity wards need the essential knowledge and skills of immediate newborn care to reduce neonatal morbidity and mortality. Continuous training and updates on standardized immediate newborn care can improve the quality of newborn care in all healthcare facilities. Faculty and students from the University of Rwanda, MScN Neonatal Program, could conduct pre-test and posttest interventional studies similar to the one in Vietnam [25] to assess sustained knowledge and assist with updating the WHO protocols and policies at selected hospitals.

\section{CONCLUSION}

The study was conducted with BAs and 171 newborns at a district hospital in Rwanda. Birth preparedness was associated with the stability of the newborn. Though a significant number of newborns in the study population were stable within the first hour after birth, a gap was identified in the provision of immediate newborn care per the current WHO protocol. To achieve the SDGs targets for ending preventable newborn and maternal deaths, BAs must have the competencies, supplies, and equipment to provide immediate newborn care interventions to enable implementation and sustain quality.

\section{Acknowledgments}

We acknowledge all the new mothers, their newborns, and the birth attendants who participated in this study and contributed to its success. We are also grateful to neonatal colleagues Richard Nsengiyumva, MScN, and Joella Mukashyaka MScN for providing help with the manuscript process. I express my deepest appreciation to my colleague Mrs Deborah Mukamuhirwa for assisting with Mendeley and reviewing my manuscript and Emmanuel Bikolimana for help with statistics.

This article is published open access under the Creative Commons Attribution-NonCommercial NoDerivatives (CC BYNCND4.0). People can copy and redistribute the article only for noncommercial purposes and as long as they give appropriate credit to the authors. They cannot distribute any modified material obtained by remixing, transforming or building upon this article. See https://creativecommons.org/licenses/by-ncnd/4.0/ 


\section{REFERENCES}

1. World Health Organization. Newborns: reducing mortality [Internet]. 2019 Sep. Available from: https://www.who.int/newsroom/fact-sheets/detail/newbornsreducing-mortality.

2. UNICEF. Levels \& Trends in Child Mortality Estimates developed by the UN Inter-agency Group for Child Mortality Estimation [Internet]. New York; 2019. Available from: https://www.unicef.org/media/60561/file/ UN-IGME-child-mortality-report-2019.pdf.

3. WHO. Recommendations on newborn health: approved by the WHO Guidelines Review Committee. Word Heal Organ [Internet]. 2017;(May):1-28. Available from: https://www.who.int/maternal_child_adole scent/documents/newborn-healthrecommendations/en/\%0Ahttp:/ apps.who .int/iris/bitstream/handle/10665/259269 /WHO-MCA-17.07.

4. WHO; UNICEF; UNFPA. Pregnancy, Childbirth, Postpartum, and Newborn Care: A guide for essential practice. In: Integrated Management of Pregnancy and Child Birth [Internet]. 3rd ed. Healthy Newborn Network; 2015. p. 54-67. Available from: https://www.healthynewbornnetwork.org/r esource/pregnancy-childbirth-postpartumand-newborn-care-a-guide-for-essentialpractice-3rd-edition/.

5. De Graft-Johnson J, Vesel L, Rosen HE, Rawlins B, Abwao S, Mazia G, et al. Crosssectional observational assessment of quality of newborn care immediately after birth in health facilities across six subSaharan African countries. BMJ Open [Internet]. 2017;7(3). Available from: https: / / pubmed.ncbi.nlm.nih.gov/2834819 $4 /$.

6. Conde-Agudelo A D-RJ. Kangaroo mother care to reduce morbidity and mortality in low birthweight infants (Review). NIH [Internet]. 2016; Available from: https://pubmed.ncbi.nlm.nih.gov/2755252 $1 /$.

7. Neovita. Study Group.Timing of initiation, patterns of breastfeeding, and infant survival : prospective analysis of pooled data from three. Lancet Glob Heal [Internet]. 2016;4(4):266-75. Available from: https://pubmed.ncbi.nlm.nih.gov/2701331 $3 /$.

8. Rwanda MoH. Neonatal protocols. 2015;83. Available from: http://www.childrenshospital.org/ /media /research-andinnovation/divisions/newbornmedicine/neonatalprotocolsrwanda.ashx.

9. Rwanda Biomedical Center. Neonatal indicators, Jan - Apr 2019. 2019.

10. Rwanda Demographic Health Survey. RDHS [Internet]. Kigali, 2016. Available from: fhttps://dhsprogram.com/pubs/pdf/FR31 6/FR316.pdf.

11. Khurmi, M.S., Sayinzoga, F., Berhe, A., Bucyana, T., Mwali, A.K., Manzi E and M. Newborn Survival Case Study in Rwanda Bottleneck Analysis and Projections in Key Maternal and Child Mortality Rates Using Lives Saved Tool ( LiST ). Int J MCH AIDS, 6(2), [Internet]. 2017;6(2):93-108. Available from:

https://pubmed.ncbi.nlm.nih.gov/2936788 $6 /$.

12. Musafili, A., Persson, L.Å., Baribwira, C., Påfs, J., Mulindwa, P.A. and Essén, B. 2017. Case review of perinatal deaths at hospitals in Kigali, Rwanda: perinatal audit with application of a three-delays analysis. BMC [Internet]. 2017;17(1):1-13. Available from: https://pubmed.ncbi.nlm.nih.gov/2828419 $7 /$.

13. World Health Statistics, 2018. Monitoring health for the SDGs, sustainable development goals [Internet]. Geneva; 2018. Available from: https://apps.who.int/iris/bitstream/handl e/10665/272596/9789241565585eng.pdf?sequence $=1 \&$ is Allowed $=y$.

14. Silvestre MAA, Mannava P, Corsino ANN, Capili DS, Calibo AP, Tan F, et al. Improving immediate newborn care practices in Philippine hospitals : impact of a national quality of care initiative $2008-2015$. Int J Qual Heal Care [Internet]. 2018;30(7):53744. Available from: https://academic.oup.com/intqhc/article/ 30/7/537/4957982.

15. Thomas MR, Yoxall CW, Weeks AD, Duley L. 
Providing newborn resuscitation at the mother's bedside: Assessing the safety, usability and acceptability of a mobile trolley. BMC Pediatr [Internet]. 2014;14(1):2-7. Available from: https: / / bmcpediatr.biomedcentral.com/tra ck/pdf/10.1186/1471-2431-14-135.

16. Lissauer T, Fanaroff AA, Miall L FF. Neonatology at a Glance [Internet]. 3rd ed. 2015. 224 p. Available from: https://bok.africa $/$ book $/ 2620030 / 89292 d$ ?dsource= recommend\&regionChanged $=$.

17. Gobezie WA, Bailey P, Keyes E, Ruano AL, Teklie $\mathrm{H}$. Readiness to treat and factors associated with survival of newborns with breathing difficulties in Ethiopia. BMC Health Serv Res. 2019;19(1):1-10.

18. Shikuku DN, Milimo B, Ayebare E, Gisore P, Nalwadda G. Practice and outcomes of neonatal resuscitation for newborns with birth asphyxia at Kakamega County General Hospital, Kenya: A direct observation study. BMC Pediatr [Internet]. 2018;18(1):1-11. Available from: https://www.ncbi.nlm.nih.gov/pmc/article s/PMC5953400/pdf/12887_2018_Article_1 127.pdf.

19. Uwingabire F, Gowan M. Birth asphyxia at a district hospital in Kigali, Rwanda. Rwanda J Med Health Sci. 2019;2(2):96-104.

20. Madhavanprabhakaran GK, Wittmann AL, Aldughaishi T, Thomas DS. Knowledge and practice of umbilical cord clamping among maternity care providers. JMRH [Internet]. 2017;3(6):1311-8. Available from: https://pdfs.semanticscholar.org/41af/fdf9 d1e052259679f454f845ad90c8c5f7fd.pdf.

21. Bee M, Shiroor A, Hill Z. Neonatal care practices in sub-Saharan Africa: a systematic review of quantitative and qualitative data. $J$ Heal Popul Nutr [Internet]. 2018;37(9):1-12. Available from: https://www.ncbi.nlm.nih.gov/pmc/article s/PMC5902879/pdf/41043_2018_Article_1 41.pdf.

22. King TL, Brucker MC OKVM. Varney's Midwifery Fifth Edition. Jones Bartlett Learn [Internet]. 2015; 1200. Available from: https://www.amazon.com/VarneysMidwifery-Tekoa-L-King/dp/1284160211.

23. Singh K, Khan SM, Carolina N. The importance of skin-to-skin contact for early initiation of breastfeeding in Nigeria and Bangladesh. J Glob Health. 2017;7(2):1-9.

24. Safari K, Saeed AA, Hasan SS, Moghaddambanaem $L$. The effect of mother and newborn early skin-to-skin contact on initiation of breastfeeding, newborn temperature and duration of third stage of labour. Int Breastfeed J [Internet]. 2018;13(32):1-8. Available from: https://www.ncbi.nlm.nih.gov/pmc/article s/PMC6048813/pdf/13006_2018_Article_1 74.pdf.

25. Tran HT, Mannava P, Murray JCS, Nguyen PTT, Tuyen LTM, Anh TH, et al. Early Essential Newborn Care is associated with reduced adverse neonatal outcomes in a tertiary hospital in Da Nang, Viet Nam: A Pre- Post- Intervention Study. Lancet [Internet]. 2018;6:51-8. Available from: https://europepmc.org/backend/ptpmcren der.fcgi?accid=PMC6537584\&blobtype $=$ pdf.

26. WHO. Every newborn [Internet]. 2014. Available from: https://www.who.int/maternal_child_adole scent/topics/newborn/enap_consultation/ en/. 